\section{DANZA Y EDUCACIÓN NOBILIARIA EN ELSIGLOXVIIII: EL MÉTODO DE LA ESCUELA DE BAILE EN EL REAL SEMINARIO DE NOBLES DE MADRID}

\section{DIANA CAMPÓO SCHELOTTO}

Centro Superior de Música del País Vasco-MUSIKENE

Resumen: El artículo presenta la edición y contextualización histórica de un manuscrito inédito, datado en septiembre de 1800 , en el que se establecen los principios para la enseñanza de la danza en el Real Seminario de Nobles de Madrid. Las instrucciones que contiene evidencian la práctica de la danza cortesana francesa, introducida en España con la llegada de Felipe V. El texto pone de manifiesto la función que en la época se atribuía al aprendizaje de la danza dentro del contexto más amplio de la educación aristocrática, como expresión del decoro nobiliario de quienes debían distinguirse por su nacimiento y estaban destinados a ocupar los más altos cargos políticos en la monarquía.

Palabras clave: Danza, Cortesanía, Real Seminario de Nobles de Madrid, Educación de la nobleza, Felipe V, Carlos IV, Escuela Bolera.

Abstract: This article presents the edition and historical context of an unpublished manuscript from 1800 , belonging to the Real Seminario de Nobles de Madrid, which establishes the principles that had to be observed by the dancing masters of this institution.

The instructions contained in the document show the adoption of the French dancing style introduced in Spain with the arrival of Philip V. The dancing masters had to teach the principles of this art in the wider context of the education of manners to those who had to distinguish themselves because of their noble origin and who were destined to occupy the higher political offices in the monarchy.

Keywords: Dance, Courtliness, Real Seminario de Nobles de Madrid, Education of the nobility, Philip V, Charles IV, Bolero School.

Résumé: Ĺarticle présente l'édition, et la mise en contexte historique d'un manuscrit inédit daté de septembre 1800, où sont établis les principes pour l'enseignement de la danse au Séminaire Royal des Nobles de Madrid. Les instructions qu'il contient mettent en évidence la pratique de la danse courtisane française, introduite en Espagne à I'arrivée de Philippe V. Le texte souligne le rôle que l'on attribuait à l'époque à l'apprentissage de la danse dans le contexte plus large de l'éducation aristocratique, en tant qu'expression de la dignité nobiliaire de ceux qui devaient se distinguer de par leur naissance et étaient destinés à occuper les postes politiques les plus élevés de la monarchie.

Mots-clés: Danse, Art du courtisan, Séminaire Royal des Nobles de Madrid, Éducation de la noblesse, Philippe V, Charles IV, École Bolera.
Dance and education of the nobility in the seventeenth century: the Método of the dance school in the Real Seminario de Nobles of Madrid

Danse et éducation nobiliaire au XVIIlème siècle: la Méthode de l'école de danse au Séminaire Royal des Nobles de Madrid 


\section{Metodo que se ha de observar en la escuela de Bayle de este Real Seminario}

El 18 de septiembre de 1800 , el regente de estudios del Real Seminario de Nobles de Madrid, Manuel de Valbuena, escribía a Josef y Antonio Rivas, maestros de baile de esa institución para remitirles un documento titulado Metodo que se ha de observar en la escuela de Bayle de este Real Seminario, (en adelante lo citaré como Método) ${ }^{1}$. En su carta, Valbuena les ordenaba atenerse a este método de manera constante, $y$ sin realizar ninguna modificación.

El Método, manuscrito por el propio Valbuena, establece desde sus primeros párrafos la relación existente entre el aprendizaje de la danza y el de los aspectos gestuales de las buenas maneras. Se inscribe por ello dentro de una tradición europea vigente durante la Edad Moderna, a la que pertenecen algunos de los más importantes tratados de danza destinados a la educación de la nobleza publicados en Europa durante ese período.

El texto está organizado en cuatro bloques temáticos: en el primero se definen las obligaciones de los maestros de baile, como transmisores del lenguaje gestual de la cortesía, implícito en los códigos del arte de la danza. La segunda parte comprende el método de enseñanza propiamente dicho, organizado en nueve lecciones para enseñar los pasos básicos de la danza a la francesa. La tercera parte incluye la explicación de la forma de bailar el minué, con sus reglas para dar las manos y mover la cabeza y los brazos. El texto concluye con una Advertencia sobre las cortesías, que remite a los principios fundamentales que regían el lenguaje corporal en general, y específicamente el de la danza para la nobleza cortesana.
El presente artículo tiene la finalidad de presentar el Método, situándolo en el contexto de la enseñanza de la danza para la educación de la nobleza durante el Antiguo Régimen, y especialmente la que se llevó a cabo en el Real Seminario de Nobles de Madrid.

\section{Las buenas maneras en los tratados de danza europeos del siglo XVIII}

En su primera sección, el Método establecía claramente las obligaciones de los maestros de danza del Real Seminario de Nobles. El aula de danza era el espacio para el aprendizaje de los códigos gestuales propios de la comunicación en sociedad, vigentes entre la nobleza. El aprendizaje de gestos como el caminar, saludar, quitarse el sombrero, realizar las reverencias conforme al status de la persona a la que se dirigían, y en general comportarse con decoro y distinción eran destrezas que actuaban como elemento de distinción estamental, a la vez que habilitaban a los seminaristas para el trato en sociedad y para la vida política.

La vinculación entre danza y buenas maneras es una constante en los tratados publicados en toda Europa durante el siglo XVIII, como Le Mâitre à Danser de Pierre Rameau (1725), The Art of Dancing de Kellom Tomlinson (Londres, 1735), o Trattato del Ballo Nobile de Giambatista Dufort (Nápoles, 1728). En España debe mencionarse Reglas útiles para los aficionados a danzar, publicado por Bartolomé Ferriol y Boxeraus en Nápoles, Capua y Cádiz en 1745, quien afirmaba que "lleva esta habilidad un mote de hombre de Corte y que denota la cuna, la educación, y la disposición del sujeto". La destreza al danzar constituía un elemento de distinción estamental, pues

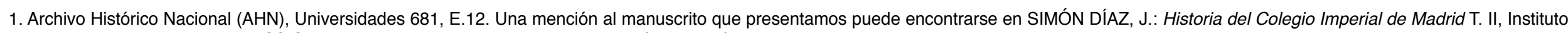
de Estudios Madrileños, Madrid, CSIC, 1952, p. 207, aunque el autor no transcribió ni analizó su contenido. 
permitía a damas y caballeros demostrar su status nobiliario sin necesidad de exhibir sus patentes de nobleza. Por el contrario, "...los errores de su execucion, desgarbo en los movimientos, falta de enseñanza, noticia y proporción, harán ser mal mirado a qualesquiera que en festiva Assamblea de muestra de una rústica indole, o crianza indiscreta, y menos política”2.

Especial mención merece The Rudiments of Genteel Behavior, de Francis Nivelon (Londres, 1737). Publicado por un maestro de danza francés, no trataba de danza, sino que enseñaba de manera detallada la forma en que las personas distinguidas debían mantenerse de pie, sentados, caminar o pasear, quitarse y ponerse el sombrero, hacer las reverencias ante personas de diferente rango, entregar y recibir objetos, esperar una respuesta, saludar al llegar o al despedirse, las mismas acciones cuyo aprendizaje quedaba a cargo de los maestros de baile según el Método del Real Seminario del Nobles ${ }^{3}$.

\section{Danza y cortesanía en el Real Seminario de Nobles de Madrid: 1725-1808}

La danza formó parte de las enseñanzas impartidas en el Real Seminario de Nobles de Madrid desde su fundación por Felipe V en 1725. Dependiente del Colegio Imperial de la Compañía de Jesús, e inspirado en el modelo del colegio Louis-le-Grand de París, su finalidad era la de educar a los vástagos de la nobleza para desempeñar las funciones tradicionalmente atribuidas a este estamento: el gobierno de sus casas como pater familias, y el servicio al rey en los ámbitos político y militar ${ }^{4}$. Como institución orientada a la educación nobiliaria, prestaba una especial atención a la formación de sus alumnos en la cortesanía, término empleado en España desde el siglo XVI, y vigente aún en el XVIII para definir el comportamiento cortés, prudente y discreto propio de quienes frecuentaban la corte, y que por extensión también se aplicaba a cualquiera que se distinguiese por su mesura y afabilidad, fuese cual fuese su status social ${ }^{5}$. El concepto de cortesanía resumía un conjunto de cualidades morales, destrezas sociales y prácticas políticas dentro de las cuales la danza cumplía la función de educar los gestos corporales según los principios del decoro e interactuar en el ámbito de la sociabilidad aristocrática.

En la historia del Real Seminario durante el siglo XVIII pueden diferenciarse dos etapas, delimitadas por la expulsión de la Compañía de Jesús por el rey Carlos III en 1767. Así, el primer período comprende desde el momento de su fundación en 1725 hasta 1767, mientras que el segundo cuenta desde ese momento hasta su cierre en 1808 como consecuencia de la invasión de

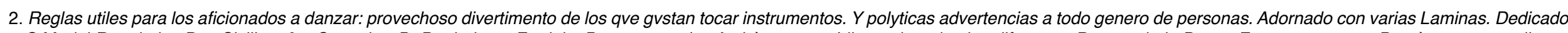

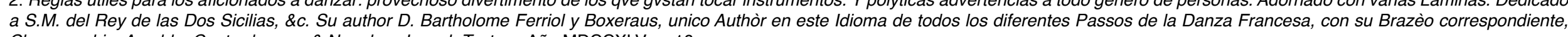
Chorographia, Amable, Contradanzas, \&.Napoles, Joseph Testore, Año MDCCXLV, p. 16.

3. NIVELON, F.: The Rudiments of Genteel Behavior. Reimpresión en facsímil de la única edición de 1737, Londres, Paul Holberton Publishing, 2003.

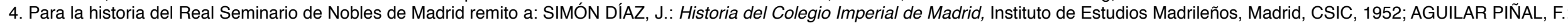

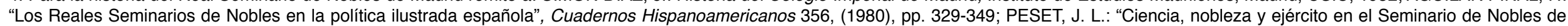

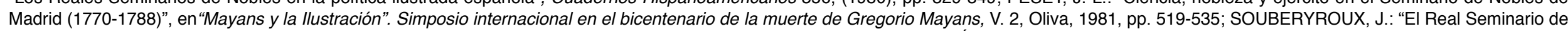

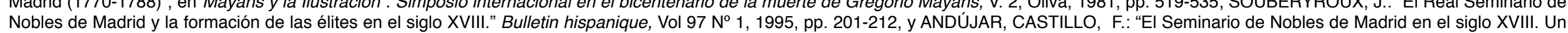
estudio social. Cuadernos de Historia Moderna. Anejos, 2004, III, pp. 201-225.

5. ÁLVAREZ-OSSORIO ALVARIÑO, A.: "Corte y cortesanos en la monarquía de España”, en QUONDAM, A. y PATRIZI, G. (eds.): Educare il corpo, educare la parola, Roma, Bulzoni Editore, 1998, pp. 297-365. 
Madrid por las tropas napoleónicas. Las consecuencias de la expulsión de los jesuitas para la enseñanza de la danza en el Seminario no se hicieron notar hasta 1770.

\subsection{La enseñanza de la danza en el Real Seminario de Nobles entre 1725 y 1767}

Las primeras Constituciones del Real Seminario, aprobadas por el rey en 1730 establecían que los seminaristas debían estudiar las "facultades y ciencias que más adornan a la nobleza", como gramática, retórica, poesía, lengua francesa, italiana y griega, matemática y geografía, así como habilidades de caballeros: danza, música, esgrima y equitación. La enseñanza de estas destrezas estaba organizado de manera precisa por el rector del Seminario, que la encomendaba a maestros seculares ${ }^{6}$. El ejercicio de las habilidades caballerescas constituía un elemento de distinción estamental, por ser consideradas un entretenimiento apropiado para la ilustre condición de los seminaristas, a diferencia de los juegos vulgares practicados por otros niños ${ }^{7}$.

El estudio obligatorio de la danza por parte de los alumnos del Real Seminario de Nobles es consecuente con el valor que la pedagogía jesuítica atribuía a su práctica ${ }^{8}$. Por otra parte, la necesidad de este aprendizaje era reconocida desde antiguo por la nobleza española. Ya en 1592, el conde de Portalegre escribía una célebre instrucción dirigida a su hijo, con consejos para comportarse apropiadamente en la corte. Decía que "dançar con gracia y soltura [...] "aprovecha para estar y caminar de buen ayre y hazer reverencias sin desgracias y assí viene a ser más necessario de lo que parece, y también lo es en qualquier tiempo que huviere damas" ${ }^{\prime \prime}$. Se refería de esta manera al imprescindible dominio del lenguaje corporal necesario para participar en la vida social cortesana, para la representación de la distinción social según los principios del decorum clásico, y para actuar adecuadamente en las funciones ceremoniales y diplomáticas que los nobles desempeñaban al servicio del rey ${ }^{10}$. La recomendación que el conde de Portalegre hacía a su hijo acerca de la práctica de la danza se hacía eco de una larga tradición, cuyos principios fueron enunciados por Baldassare Castiglione en II Libro del Cortegiano, obra que ejercería una notable influencia entre la nobleza española ${ }^{11}$.

Este ideal cortesano tendría un impacto notorio en el desarrollo de la escuela española de danza del siglo XVII. El término sprezzatura fue empleado por Castiglione para indicar la manera de comportarse con gracia en todas las acciones, imprescindible para actuar con éxito en la corte. Fue traducido al

6. Constituciones de el Real Seminario de Nobles fundado en el Colegio Imperial de la Compañía de Jesus de Madrid. Año 1730 por el Señor Don Phelipe Quinto [...], Madrid, 1730 , p. 13.

7. Ibíd., p. 42

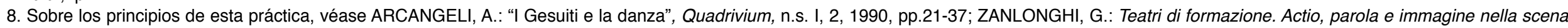
gesuitica del Sei-Settecento a Milano, Milán, Vita e Pensiero, 2000.

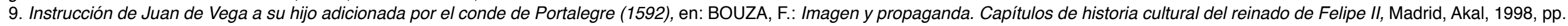
229-230.

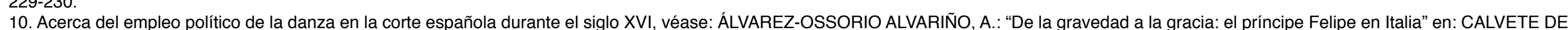

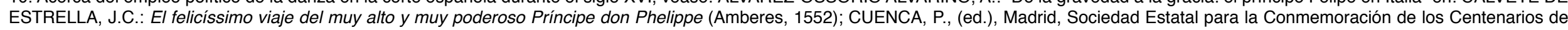
Felipe II y Carlos V, 2000. pp. LXXVII-CXIV.

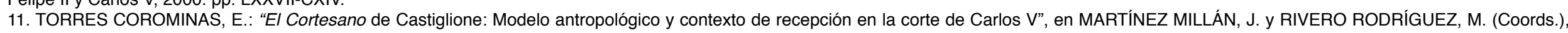
Centros de poder italianos en la Monarquía Hispánica (siglos XV-XVIII), Madrid, Polifemo, 2010, pp. 1183-1234. 
castellano por Juan Boscán en 1534 como descuido, desprecio o despejo, y opuesto al vicio de la afectación, en la que sería la primera traducción europea del Cortegiano ${ }^{12}$. El descuido consistía en actuar con una aparente naturalidad, que ocultaba el esfuerzo, hacía imperceptibles las dificultades de cualquier acción, y simulaba renunciar a la búsqueda de la perfección. En el caso de la danza, el descuido se mostraba en que "... en el danzar un solo paso o un solo movimiento, que se haga con buen aire y no forzado, en la misma hora descubre el saber de quien danza ${ }^{13}$." Esta estudiada facilidad en las acciones, al rechazar la exageración en el comportamiento, era considerada en la época una expresión de la mesura, propia de quienes actuaban conforme a las pautas de una segunda naturaleza adquirida mediante la educación cortesana, por lo que comprendía una valoración moral, estética y social ${ }^{14}$.

Los conceptos opuestos de descuido y afectación serían incorporados por Juan de Esquivel Navarro en sus Discursos sobre el arte del Dançado (1642), en que recogía las enseñanzas de Antonio de Almenda, maestro de danza de Felipe IV. Esquivel afirmaba que la danza era "un descuido cuidadoso", en la que el cuerpo debía llevarse "bien derecho sin artificio, con mucho descuido, del mismo modo que se lleva por la calle sin endereçarle mas de aquello que su natural le dà [...] Por que la afectación y presuncion es cosa con que se desluze todo quanto se obra bien"15. Los ojos debían llevarse serenos y a media altura, "con descuido", y los brazos relajados a los lados del cuerpo, evitando los movimientos exagerados.

A finales del siglo XVII, la escuela francesa de la danza entraría en la corte española, aunque de manera efímera. Los maestros de danza que acompañaron a María Luisa de Orléans al contraer matrimonio con Carlos Il en 1679, así como los músicos franceses de la reina fueron enviados de regreso a Francia un año más tarde ${ }^{16}$. La introducción de esta escuela de manera permanente se inició con la llegada de Felipe $V$, a través de Nicolás Fontón, Michel Gaudrau y Sebastián Christiani de Scio, maestros de danza de las reinas María Luisa Gabriela de Saboya e Isabel de Farnesio, y de los infantes nacidos de ambos matrimonios. Todos ellos disfrutaron de la protección de los monarcas, y de una posición privilegiada en la corte ${ }^{17}$.

Si en un primer momento la difusión de la danza a la francesa se debió a la labor de los maestros de danza que servían a los reyes y a la nobleza, más tarde contribuiría a ello la publicación de los tratados publicados por Pablo Minguet e Yrol a partir de 1737, y el de Bartolomé Ferriol antes mencionado. Coincidiendo con el auge de los bailes públicos promovidos por el conde de Aranda desde 1767, se inició la publicación periódica de colecciones

12. CASTIGLIONE, B.: El Cortesano, Madrid, Cátedra, 1994, I, 27, pp. 143-147.

13. Ibidem, I, 28, p. 149: "Asimismo en el danzar un solo paso o un solo movimiento, que se haga con buen aire y no forzado, en la misma hora descubre el saber de quien danza."

14. QUONDAM, A.: "La 'Forma de vida'. Apuntes para el análisis del discurso cortesano", en QUONDAM, A.: El Discurso Cortesano, Madrid, Polifemo, 2013 , pp. $23-27$.

15. ESQUIVEL NAVARRO, J.: Discursos sobre el arte del Dançado y sus excelencias, reprobando las acciones deshonestas, Sevilla, 1642, p. 21v.

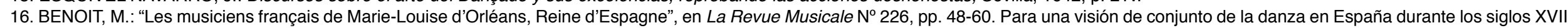
y XVIII, véase ESSES, M.: Dance and Instrumental Diferencias in Spain during the 17th and Early 18th Centuries, New York, Pendragon Press, Stuyvesant, 1992.

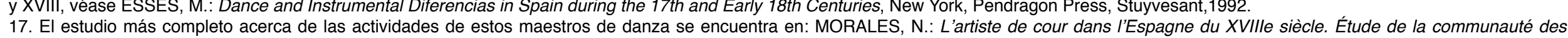

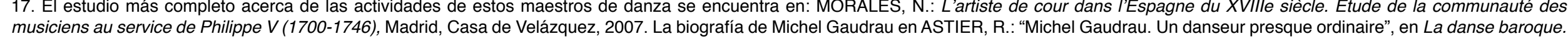

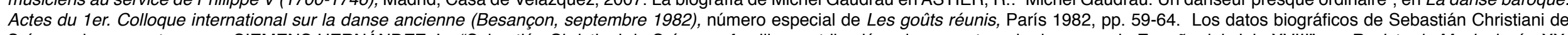

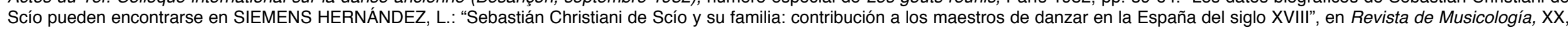
1, 1997, pp. 323-330. 
de contradanzas que pondrían el aprendizaje de esta destreza al alcance de todos aquellos capaces de leer ${ }^{18}$. Otra de las vías por las que la danza francesa se difundió en la sociedad fue a través de las instituciones dedicadas a la educación de la nobleza, como el Real Seminario de Nobles de Madrid.

La vinculación entre danza, buenas maneras y sociabilidad aristocrática era común a la escuela francesa y a la española, que coexistieron durante más de medio siglo. El primer período de existencia del Real Seminario se caracterizó por la enseñanza de forma paralela de ambos estilos, algo semejante a lo que ocurría con la enseñanza musical, para la cual existía un maestro de violín, instrumento por excelencia de los maestros de danza franceses, y uno de vihuela (guitarra), representativo en la época de la música y la danza españolas ${ }^{19}$. Los nombres de los maestros de danza de este período están recogidos en la siguiente tabla:

\begin{tabular}{|c|c|c|}
\hline \multicolumn{3}{|c|}{$\begin{array}{l}\text { Maestros de danza en el Real Seminario de Nobles de Madrid } \\
\qquad 1730-1770\end{array}$} \\
\hline Francisco de Torres $^{20}$ & $\begin{array}{l}i-1730 \\
1730-?\end{array}$ & $\begin{array}{l}\text { Danza española } \\
\text { Danza francesa }\end{array}$ \\
\hline Martín Ximeno ${ }^{21}$ & $1730-1759$ & Danza española \\
\hline Andrés de la Roca de Tolis ${ }^{22}$ & $1756-1770$ & Danza francesa \\
\hline Bernardo Bottini ${ }^{23}$ & $\begin{array}{l}\text { Al menos desde } \\
1757-1770\end{array}$ & Danza francesa \\
\hline Juan Francisco Clemente ${ }^{24}$ & $1766-1770$ & Danza española y francesa \\
\hline Joseph Marset ${ }^{25}$ & $1767-1779$ & Danza francesa \\
\hline
\end{tabular}

Entre las obligaciones de los maestros de danza de este período estaba la de preparar a los seminaristas para participar en las representaciones teatrales que se realizaban en el Real Seminario. Desde el siglo XVI, la danza formaba parte de las representaciones teatrales de los colegios jesuitas españoles. Estas representaciones se planteaban como ejercicios prácticos de retórica, en los que se desplegaban todas las cualidades de la elocuencia verbal y

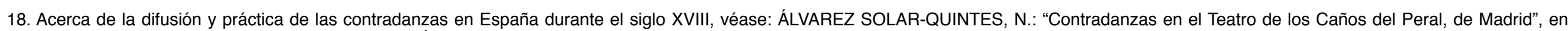

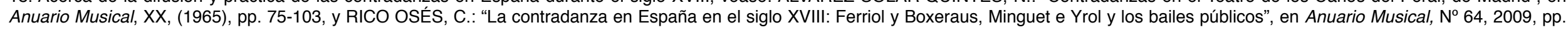

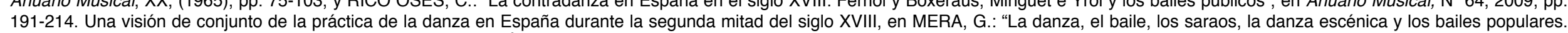

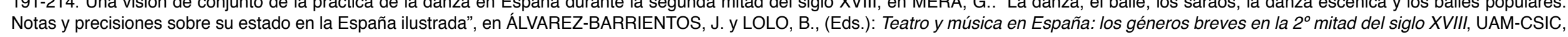
Madrid 2008, pp. 459-479.

19. ESSES, M.: Dance and instrumental diferencias... op. cit., pp. 113-142.

20. Libro de gasto ordinario y extraordinario del Real Seminario de Nobles, AHN, Universidades, L.1305, p. 60v.

21. Ibid. p. 60v. y Archivo General de Simancas (AGS), GyJ, L.969. (s.n.).

22. Era hijo del maestro de danza francés André de la Roche, a quien sucedió en este oficio en la Casa de Caballeros Pajes del Rey. Archivo General de Palacio (AGP), EP C889 E48.

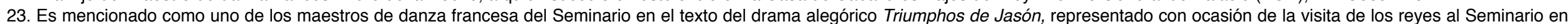

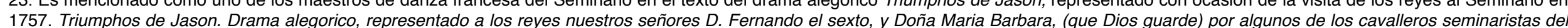
Real Seminario de Nobles de Madrid, [...] Madrid, 1757, p.40.

24. Era maestro de danza española, francesa, "bailes ingleses y alemandas". AGS, GyJ, L.969 (s.n.)

25. AGS, GyJ, L.969 (s.n.). 
gestual, dentro de un espectáculo de contenido moralizante y educativo. En los entreactos se acostumbraba intercalar breves piezas jocosas cuyo principal objetivo era el de despertar el interés de los espectadores y rebajar la tensión dramática. En los finales, los pasajes musicales y danzados actuaban como conclusión de la acción dramática, dotándolos de una mayor intensidad emocional $^{26}$.

Los espectáculos más importantes organizados en el Real Seminario de Nobles de Madrid durante este período continuaban con esta tradición. Seguían el calendario escolar que se iniciaba con la inauguración del curso el 18 de octubre, día de San Lucas. Además, se celebraban las fiestas de la liturgia cristiana, fiestas particulares en honor de algún santo, y las visitas de importantes personas reales o eclesiásticas ${ }^{27}$. También está documentada la celebración de conclusiones públicas, en las que los seminaristas mostraban sus progresos.

En estas ocasiones, además del rey, asistía la más alta nobleza, miembros de los consejos reales y embajadores. Los maestros de danza preparaban a los seminaristas para realizar diversas danzas, a la española y a la francesa, que se integraban dentro del espectáculo teatral. A diferencia de lo que sucedía en Louis-le-Grand, los propios seminaristas representaban todos los papeles, sin que conste la participación de actores o bailarines profesionales ${ }^{28}$. Por el contrario, existe constancia de pagos a músicos, tanto para la composición de las obras, como para la asistencia a ensayos y funciones.

\subsection{La enseñanza de la danza en el Real Seminario de Nobles entre 1770 y 1808}

Después de la expulsión de la Compañía de Jesús en 1767, el Real Seminario de Nobles pasó un período de crisis durante el cual número de seminaristas descendió de ciento tres a diecinueve, lo que fue atribuido por las autoridades a la creencia general de que sólo los jesuitas podían proporcionar una buena educación a la nobleza. Para remediar esta situación, en 1770 fue nombrado como director el marino Jorge Juan, quien reformularía el plan educativo, reforzando la enseñanza de las materias científicas necesarias para la formación de marinos y militares ${ }^{29}$. Se mantuvieron, sin embargo, los fundamentos de la formación nobiliaria, en sus aspectos morales, religiosos y políticos, lo que implicaba la continuidad en la enseñanza de las habilidades caballerescas.

La reforma, no obstante, supuso la supresión de la escuela española. Todos los maestros de danza fueron jubilados salvo Joseph Marset, quien ocuparía la plaza de asistente de Esteban Rossell, antiguo profesor de violín, nombrado primer maestro de danza. También se suprimiría la enseñanza de la guitarra, quedando dos maestros de violín ${ }^{30}$. Esteban Rossell sería designado en 1781 como maestro de danza de la familia real, por lo que su competencia profesional queda fuera de duda. Desde este oficio ejercería su influencia en los nombramientos de otros maestros del Real Seminario ${ }^{31}$.

26. MENÉNDEZ PELÁEZ, J.: Los Jesuitas y el Teatro en el Siglo de Oro, Oviedo, Universidad de Oviedo, 1995, pp.59-91. 27. Ibid., pp. 89-91.

28. ASTIER, R. y PEARCE, D.: "Pierre Beauchamps and the Ballets de Collège”, Dance Chronicle, Vol. 6, № 2 (1983), p. 141.

29. PESET, J. L.: "Ciencia, nobleza y ejército...", op. cit., pp. 520-522.

30. AGS, GyJ, L. 969 (s.n.)

31. AGS, GyJ, L. 969. 


\begin{tabular}{|c|c|c|}
\hline \multicolumn{3}{|c|}{$\begin{array}{l}\text { Maestros de baile en el Real Seminario de Nobles de Madrid } \\
\qquad 1770-1808\end{array}$} \\
\hline Esteban Rossell & $\begin{array}{l}i-1770 \\
1770-1781\end{array}$ & $\begin{array}{l}\text { Maestro de violín } \\
1^{\circ} \text { maestro de baile }\end{array}$ \\
\hline Joseph Marset & $\begin{array}{l}1767-1770 \\
1770-1779^{\star}\end{array}$ & $\begin{array}{l}\text { Maestro de baile } \\
2^{\circ} \text { maestro de baile }\end{array}$ \\
\hline Gerónimo Cavañez & $\begin{array}{l}i-1781 \\
1781-1790^{\star \star}\end{array}$ & $\begin{array}{l}2^{\circ} \text { maestro de baile } \\
1^{\circ} \text { maestro de baile }\end{array}$ \\
\hline Juan Gamot & $1781-1793^{\star \star}$ & $2^{\circ}$ maestro de baile \\
\hline Rafael Rivas & Oct.-Dic.-1783** & Maestro de baile \\
\hline Jacinto Carrera & $1783-1798(\dagger)$ ** & Maestro de baile \\
\hline Pedro Pizzi & $1786-1788(†)$ & Maestro de baile \\
\hline Esteban Peré & $1788-1800(\dagger)$ & Maestro de baile \\
\hline Domingo Belluzzi & $1791-1793$ & Maestro de baile \\
\hline Lorenzo Soto & 1798-1799 (despedido) & Maestro de baile \\
\hline Josef Rivas & $1799-1808$ & $1^{\circ}$ maestro de baile \\
\hline Antonio Rivas & $1800-1808$ & $2^{\circ}$ maestro de baile \\
\hline Josef González & $\begin{array}{l}1791-1802 \\
1802-1808\end{array}$ & $\begin{array}{l}\text { Supernumerario } \\
3^{\circ} \text { maestro de baile }\end{array}$ \\
\hline
\end{tabular}

* Marset fue elegido como segundo maestro de baile por Esteban Rossell, cuando éste fue promovido de maestro de violín a primer maestro de baile.

** Estos maestros fueron recomendados por Esteban Rossell después de ser nombrado maestro de baile de la familia real.

En el año 1785 se redactaría un plan de estudios para el Real Seminario que sentaría las bases de su funcionamiento para los años posteriores ${ }^{32}$. En él se establecía que se enseñaría el "Minuet y el Paspié, diversos pasos del bayle inglés, contradanzas y las figuras de estas ${ }^{33}$ ". También se señalaba expresamente la responsabilidad de los maestros de baile para enseñar a los seminaristas a andar con elegancia, quitarse el sombrero con gracia, hacer las reverencias, presentarse en público con dignidad, saludar y tomar asiento y pasearse en compañía de otros ${ }^{34}$. Empleaban en este estudio una hora y media diaria. Estas disposiciones continuarían vigentes hasta el cierre del Real Seminario en 1808, y serían recogidas en el Método.

Durante este segundo período de la historia del Real Seminario de Nobles existió una controversia acerca de la utilidad y conveniencia de la realización de representaciones teatrales. Una descripción anónima del estado de la institución posterior a la reforma de 1770 afirmaba que, aunque la danza era muy apropiada para que los jóvenes aprendiesen a tener una buena postura, a caminar, hacer las reverencias y demás acciones con elegancia, gravedad y decoro, debía impedirse que la afición al baile les distrajese de los estudios serios $^{35}$.

32. AGUILAR PIÑAL, F.: “Los Reales Seminarios de Nobles...”, op. cit., pp. 336-339.

33. AHN, Consejos, 1344, Exp. 3.

34. Ibíd

35. Ibid 
El 28 de septiembre de 1779, Mariano Surges, director del Real Seminario escribió a su superior, el Secretario de Gracia y Justicia Manuel de Roda trasladándole la propuesta de Manuel Valbuena, quien entonces era maestro de Poética y Retórica, de que los alumnos representasen en Navidades una tragedia compuesta por el propio Valbuena.

Surges afirmaba apoyar la propuesta de Valbuena, considerando conveniente que los seminaristas tuviesen esta diversión, que ya en otras ocasiones se había realizado. Según decía, las representaciones resultaban útiles pues los seminaristas adquirían, aún más que con los certámenes, soltura y buenos modales para el trato con otras personas ${ }^{36}$. Sin embargo, enviaba otra carta por vía reservada, en la que expresaba su verdadera opinión, contraria a la realización de representaciones teatrales.

La respuesta de Manuel de Roda hacía suyas las razones expresadas por Surges $^{37}$. Afirmaba que las representaciones teatrales distraían a los jóvenes del estudio y les ocupaban demasiado tiempo. Además, en vez de inspirarles amor a las letras cobraban odio al estudio serio necesario para adquirirlas. Por esto, Roda defendía la celebración de certámenes literarios, en los cuales se adquiría la soltura para hablar en público, respondiendo a las preguntas que se formulaban, en vez de recitar aquello que se había estudiado de memoria. Finalmente, los certámenes eran preferibles porque las representaciones teatrales causaban gastos excesivos al Seminario, y tampoco era justo que fuesen sufragados por las familias de los seminaristas.
Las representaciones teatrales serían suprimidas varios años más tarde, a raíz de la reorganización de los estudios y redacción de las nuevas Constituciones, aprobadas por Carlos IV el 12 de junio de $1793^{38}$. En ellas se reafirmaba la finalidad del Real Seminario de Nobles de proporcionar a éstos los fundamentos de la buena educación, que debía prepararles para el servicio al rey y a la religión. La celebración de representaciones teatrales quedaría terminantemente prohibida, al considerarse que carecían de utilidad, y sólo servían para causar gastos y otros perjuicios ${ }^{39}$. Sin embargo, esto no afectaría a la enseñanza de la danza. Para la enseñanza de las "habilidades propias de una buena educación", era voluntad del rey que hubiese un maestro de dibujo, dos de baile, dos de violín y uno de esgrima.

En julio de 1799 se redactaron una nuevas Constituciones en las que se establecía de manera precisa la distribución de todas las materias que allí se estudiaban. Dentro de una duración total de diez años, la danza se estudiaba durante los cuatro primeros cursos. A partir de ese momento, los seminaristas asistirían una vez por semana a una Academia en la cual practicaban el baile, el dibujo y la lengua francesa, con el fin de conservar sus habilidades ${ }^{40}$. La redacción del Método, sin embargo, dedicaba tres años al estudio de la danza, aunque mantenía la asistencia a las academias.

Todos los maestros del Real Seminario, incluidos los de baile, tenían la obligación de presentar periódicamente al director una relación del progreso de los alumnos a su cargo. Además, según las constituciones aprobadas en 1799, cada cuatro meses debía celebrarse un examen público, cuyo

36. AGS, GyJ, L.969.

37. Ibid.

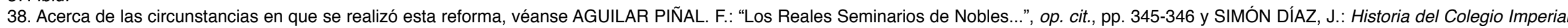
de Madrid... op. cit., V. 2, p.213.

39. AHN, Universidades, L.690 (2), Exp. 4

40. AHN, Universidades, 691(2) Exp. 5 
informe se enviaba al Secretario de Gracia y Justicia. Estos documentos nos permiten conocer algunos detalles de la manera en que se desarrollaba el aprendizaje de la danza en el Real Seminario.

El primer paso era aprender a realizar las reverencias o "cortesías", con todos los movimientos que las componían. Para poder realizarlas era preciso conocer antes las cinco posiciones de los pies de la danza francesa, explicadas en el Método. Estas posiciones se caracterizaban por emplear la rotación externa en los miembros inferiores, realizada en la articulación de la cadera. Como consecuencia, manteniendo los talones juntos, los muslos, las piernas y las puntas de los pies quedaban orientados hacia fuera, formando un ángulo de $90^{\circ}$ o más ${ }^{41}$. El Plan de Estudios y Habilidades publicado en 1785 reconocía estos fundamentos, y destinaba un maestro de baile al cometido de "imponer á los seminaristas en los principios, esto es, á volver los pies, primeras posiciones, y á tener bien derecho el cuerpo, y el pecho bien sacado, y sin afectación... ${ }^{42}$." Estos gestos formaban parte del código social de la buena educación, e iban más allá de la repetición mecánica de ciertos movimientos. Así, el maestro Antonio Ribas informaba en septiembre de 1800 que "Dn. Carlos Garnica: sabe presentarse bien y sabe todos los métodos de política y buena crianza que debe saber un caballero" ${ }^{\text {"43 }}$.

A continuación, los alumnos comenzaban a practicar el "demicupé", paso fundamental dentro de la escuela francesa ${ }^{44}$. Posteriormente, aprendían los pasos y figuras del minué, que más tarde practicaban con el acompañamiento del violín. El minué era la primera de las danzas que aprendían, en diferentes variantes: minué figurado, minué de la Corte, minué del Congó, minué escocés y minué alemandado. También aprendían el paspié (del francés passepied) una danza que se realiza con los mismos pasos del minué, aunque con algunas diferencias en cuanto al tipo de figuras y al acompañamiento musical $^{45}$. Seguidamente, aprendían a bailar las contradanzas inglesas, las francesas y las Contradanzas del Rigodón, la Gavota, la Alemanda, y el Baile inglés ${ }^{46}$.

\subsection{Entre ruptura y conservación del decoro nobiliario}

De este período datan algunos borradores que contienen interesantes reflexiones acerca de la finalidad y la manera en que debía realizarse la enseñanza de la danza en el Seminario. Las repetitivas fórmulas que establecían el aprendizaje de las cortesías y los bailes propios de personas de calidad, recogidas en las constituciones prescribían la práctica positiva de un modelo, mientras que las prohibiciones expresadas en los borradores, y eliminadas de los textos definitivos, nos permiten conocer ciertos aspectos de la práctica de la danza en la sociedad contemporánea que preocupaban a los autores de estos textos, por considerarlos indecorosos y ser contrarios al modelo de enseñanza que debía proporcionar el Seminario.

41. Esta rotación externa realizada en las caderas es denominada en dehors en la terminología actual de la danza clásica.

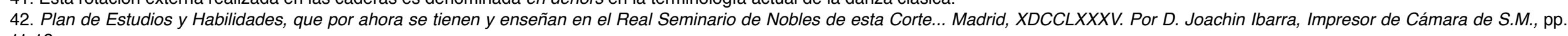
$11-12$.

43. AHN, Universidades, 676 Exp.1.

44. "Demicupé" es la transformación al castellano del término francés demi-coupé, utilizado ya por Ferriol en su tratado de 1745.

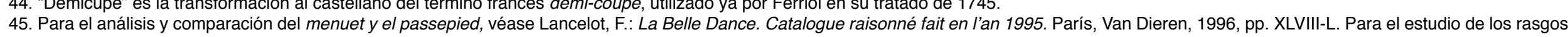

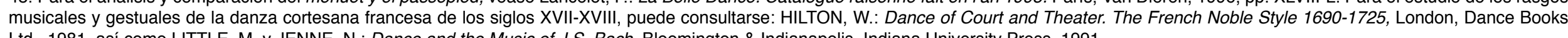
Ltd., 1981, así como LITTLE, M. y JENNE, N.: Dance and the Music of J.S. Bach, Bloomington \& Indianapolis, Indiana University Press, 1991.

46. AHN, Universidades, 676 Exp.1. 
Así, el borrador para las Constituciones de 1793, señalaba que los maestros de baile debían enseñar a los seminaristas la manera de presentarse, andar y saludar, "con natural modestia sin afectacion". Para ello sólo podrían enseñar "aquellos bailes decentes cuyo estudio contribuye a la gentileza y gallardía propia de los caballeros cristianos", quedando prohibida expresamente la enseñanza de "bailes deshonestos" cuya práctica se consideraba corruptora de la moral y contraria a la educación piadosa y cristiana ${ }^{47}$.

El autor de este texto establecía una interesante relación entre la naturalidad y falta de afectación que caracterizaban al descuido en la danza, heredero del ideal del Cortesano, con la decencia y la piedad propias de los caballeros cristianos. En ningún caso se trataba de prohibir la práctica de la danza con argumentos religiosos, sino de restringir los bailes enseñados a los seminaristas a aquellos que por la moderación de sus movimientos se consideraban conformes a las buenas costumbres.

El Plan para la educación de la nobleza y clases pudientes españolas de 1798, atribuido a Jovellanos, expresaba la misma preocupación, aunque no lo presentaba como una cuestión de piedad cristiana, sino como un problema moral relacionado con la conservación del decoro nobiliario. Además de establecer la manera en que se enseñaría el minuet, el paspié, las contradanzas y el baile inglés, prohibía expresamente la enseñanza del bolero y "otras danzas semejantes, procaces y de movimientos violentos, impropias de personas de alto rango y buenas costumbres ${ }^{48}$."
Estas observaciones acerca del decoro que debía observarse en las danzas enseñadas en el Real Seminario reflejaban la preocupación creciente de los reyes y sus ministros por la tendencia de algunos sectores de la alta nobleza a imitar las formas de comportamiento, vestimenta, conversación y bailes de los plebeyos, conocida como majismo ${ }^{49}$. Los majos (auténticos o nobles disfrazados) bailaban boleros, seguidillas y fandangos, bailes de origen popular caracterizados por sus gestos vivos, apasionados, y de un erotismo bastante explícito, sobre todo si se los compara con los pasos mesurados y elegantes del menuet o las contradanzas ${ }^{50}$.

Esta imitación de las costumbres populares era contraria a los principios mismos del sistema estamental, en el que los privilegios que distinguían a la nobleza eran la consecuencia de su supuesta superioridad moral, e implicaban la obligación de constituir un ejemplo de virtud y piedad ante el pueblo. La transgresión en la representación del rango social por parte de la aristocracia suponía una renuncia a esta obligación. Esto daría origen a una abundante normativa que pretendía poner límites a estas transgresiones, prohibiendo a los nobles adoptar la vestimenta propia de personas de baja condición, bajo pena de ser arrestados ${ }^{51}$.

La función atribuida a la danza dentro de la educación nobiliaria, y su relación con la conservación de los usos sociales y el decoro estamental queda de manifiesto con la prohibición, a través de la Real Orden del 28 de diciembre de 1799, de representar en los teatros públicos españoles obras dramáticas, música y bailes que no fuesen nacionales, con el consiguiente

47. AHN, Universidades, 690 (2) Exp. 4

48. Plan de educación de la nobleza trabajado de orden del Rey en 1798, en: ADELLAC GONZÁLEZ DE AGÜERO, M.: Manuscritos inéditos de Jovellanos. Gijón, L. Sangenís, 1915, p. 232.

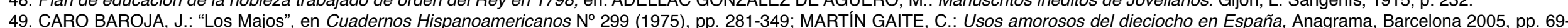

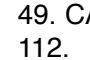

50. Recuérdese la descripción del fandango incluida en las Memorias de Casanova, en CASANOVA, G. y BARETTI, G.: Dos ilustrados italianos en la España del XVIII, Madrid, Cátedra, 2002, p. 68-69. 51. ÁLVAREZ-OSSORIO ALVARIÑO, A.: “Rango y apariencia. El decoro y la quiebra de la distinción en Castilla (ss. XVI-XVIII)", Revista de Historia Moderna No 17 (1998-1999), p. 278. 
triunfo de los bailes boleros. Esta orden se produciría en el contexto de una larga polémica que tuvo lugar durante aquellos años en el ámbito de los espectáculos teatrales, enfrentando a los defensores de los bailes españoles con los partidarios de las compañías francesas e italianas que representaban en los teatros madrileños ${ }^{52}$. A pesar de ello, la enseñanza de la danza en el Real Seminario no se vio afectada por estos cambios políticos. Los bailes boleros podrían ser adecuados para los teatros, pero la escuela francesa continuaba siendo la más apropiada para representar los principios tradicionales de la educación nobiliaria. Como muestran las relaciones de los exámenes públicos que se realizaban dos veces al año, los maestros de danza cumplieron puntualmente las órdenes recibidas. En el examen realizado en enero de 1808 , los seminaristas mostrarían públicamente su habilidad para realizar las reverencias, la manera de presentarse y manejar el sombrero, y para bailar el Minué, el Minué Alemandado, la Gavota y las Contradanzas del Rigodón ${ }^{53}$.

La "Advertencia sobre las cortesías" con la que concluía el Método aludía a la naturalidad en los gestos que distinguía a un caballero al realizar una reverencia, evitando la afectación que suponía el exceso de lentitud y de inclinación. A excepción de aquellas realizadas ante reyes y ministros, las reverencias debían hacerse sin exageraciones, "con la mayor naturalidad posible". Estas recomendaciones ponen de relieve la vigencia del ideal de comportamiento cortesano, regido por la gracia y el descuido, dentro de los códigos gestuales de la danza y de la cortesanía en España hasta finales del Antiguo Régimen.
La enseñanza de la danza en el Real Seminario de Nobles de Madrid experimentó importantes cambios a lo largo de su historia, entre los cuales debemos destacar la supresión de la escuela española en 1770, después de haber existido simultáneamente con la escuela francesa desde su fundación. La prohibición de las representaciones teatrales en 1793 puso fin a una práctica tradicionalmente aceptada como escuela de formación para la vida social y política de los nobles, por razones en las que los aspectos financieros se mezclaban con un concepto diferente de la educación. Sin embargo, el aprendizaje de la danza continuó siendo un espacio privilegiado para la adquisición de las cualidades corporales asociadas al decoro nobiliario y de las destrezas sociales y políticas propias de la cortesanía.

\section{La transcripción del documento}

El documento original está escrito a mano en cuatro folios por las dos caras sin numerar. Se ha optado por reproducir literalmente la grafía, acentuación y puntuación, así como el uso de mayúsculas y minúsculas, y la organización en párrafos. La única modificación realizada, en función de la legibilidad del texto, ha sido el desarrollo de las abreviaturas presentes en términos como "de", "que", "quando", "tiempo" "siempre" y "Señora".

52. En relación a esta controversia, véase MERA, G.: "Los ilustrados y la danza a principios del siglo XIX. Polémicas sobre la construcción de una identidad nacional frente al modelo francés", en MARTíNEZ DEL FRESNO, B. (Ed.), Coreografiar la historia europea: cuerpo, política, identidad y género en la danza. Oviedo, Universidad de Oviedo, 2011, pp. 173-197.

53. AHN. Universidades, $676(2)$. 


\section{"Metodo que se ha de observar en la escuela de Bayle de este Real Seminario}

Los Maestros de Bayle cuidaran muy particularmente de enseñar à los Caballeros Seminaristas, desde que entran en su clase el modo de presentarse desembarazadamente y con decoro delante de gentes dentro $y$ fuera de casa, tomando a su aula por exemplar de una tertulia ò concurrencia de personas de distincion. Les enseñaran el modo de quitarse el sombrero y de ponersele, despues de saludar à las personas, ya sea parados, ya de paso, à derecha, è izquierda, y asi mismo el modo de tener el sombrero debaxo del brazo, y de tomarle con la mano para ponersele, ò quitarsele.

Igualmente les instruiran en hacer todo genero de cortesias al entrar en una sala, al salir de ella, al hablar à una persona de distincion, al despedirse de ella, al recibirla en casa, y la postura que deberàn observar en pie, sentados, y andando ò paseando con sugetos iguales ó superiores.

De mismo modo les impondràn en el modo de presentarse à un Xefe, à las Personas Reales, à besar su Real mano, y el genero de inclinacion y Reverencia que deben hacer quando les hablen estas personas.

Tambien les enseñaran el modo de dar ò recibir una prenda ò alaja de otras personas. Y si es libro, papel ò cosa semejante que estas hayan de leer en su presencia, en que ademan deben esperar la respuesta. $Y$ generalmente hablando, la postura y ademan de cabeza, ojos, manos y todo el tronco del cuerpo, en que actitud le han de tener quando esten hablando con personas de respeto, ò de confianza; pues con ninguna especie de gentes, en ningùn tiempo ni lugar se deben olvidar del ademan y postura correspondientes.
El progreso que debe tener esta enseñanza desde los primeros principios, ò posiciones serà enseñar a los Caballeros Seminaristas el minue, y pasapie, el paso de las contradanzas, algo de bayle Ingles, y ultimamente un solo ò dueto serio, y algun alegro de diferentes pasos de la escuela Francesa. Se destinan tres años à esta escuela, ademas de los dias de Academia, para los que hayan salido de ella.

\section{Metodo para enseñar esta escuela con perfeccion}

Se empieza à enseñar los cinco movimientos, que se llaman posiciones, los quales sirven para que el cuerpo estè siempre en su equilibrio, y para que los pasos no salgan de la medida regular que deben tener. Se da principio: poniendo los pies juntos por los talones, mirando la punta del derecho à la derecha, y la del izquierdo à la izquierda, las rodillas firmes: y esta es la primera posicion. Luego se alargarà el pie derecho un pie de distancia del izquierdo sin moverse de la linea en que se estaba en la $1^{a}$ posicion, sin doblar las rodillas: y esta es la segunda posicion. La $3^{\mathrm{a}}$ es poner el pie derecho delante de la ebilla del izquierdo con las rodillas firmes. La $4^{\mathrm{a}}$ es adelantar el pie derecho un pie de distancia del izquierdo, esto es del de la ebilla del izquierdo adelante siguiendo la linea. La $5^{\mathrm{a}}$, es poner el tacon derecho à la punta del pie izquierdo vueltas las puntas de los pies; $y$ luego hacer lo mismo con el pie izquierdo. Todo esto ha de ser sin menear el cuerpo, sino desde el juego de la cadera abajo.

\section{Segunda leccion para el modo de marchar adelante y atras}

Se pondrà el pie derecho en la primera posicion, se marcharà con él adelante hasta la $4^{a}$ posicion, poniendo primero la punta en el suelo, y luego el talon; despues se adelantarà el izquierdo hasta la $4^{\mathrm{a}}$ cargando el cuerpo sobre el pie derecho, y observando la misma regla de sentar antes la punta que el 
talon. Para marchar hacia atras, se observarà lo mismo, à diferencia que en lugar de ser la $4^{\mathrm{a}}$ posicion adelante se harà detras: todo lo que se ha de hacer sin doblar las rodillas, ni permitir que el pie quando pase haga alguna vuelta.

\section{Tercera leccion del modo de marchar de lado à la derecha, y izquierda}

Del lado derecho, se pondrà el caballero en la $1^{a}$ posicion. Pondrà el pie derecho en la $2^{\mathrm{a}}$ y pasarà el pie izquierdo a la $5^{\mathrm{a}}$ por detras y seguira asi mismo. Del lado izquierdo cruzarà el pie derecho desde la $5^{\text {a }}$ posicion adelante à la $5^{\mathrm{a}}$ posicion atras, y harà la $2^{\mathrm{a}}$ del pie izquierdo continuarà cruzando el pie derecho à la $5^{\mathrm{a}}$ atras, y el izquierdo à la $2^{\mathrm{a}}$. Todo esto se ha de observar siempre, sin que salga nunca de alguna posicion, porque el paso seria falso, y que llegue siempre àl suelo antes la punta que el talon.

\section{Quarta leccion del doblado.}

Pondra el caballero los pies en la $1^{a}$ posicion sin entrar sobre las puntas. Doblarà las rodillas igualmente la una mirando à la derecha, y la otra à la izquierda, y doblara hasta que conozca que el cuerpo no sale de su equilibrio; luego irà subiendo poco à poco, y estando ya juntas las dos rodillas, relevarà el cuerpo sobre las puntas, teniendo las rodillas juntas, y muy firmes: despues dexarà caer los talones, y continuará doblando del modo dicho. Se ha de advertir que los doblados tienen tres tiempos, esto es doblar, subir el cuerpo, y relevarle sobre las puntas, que es un compas de musica, advirtiendo que bate el compas al subir el cuerpo.

\section{Quinta leccion, del modo de doblar adelante y atras, que se llama,} Demicupe.

Pondrà el caballero los pies en la $1^{\mathrm{a}}$ posicion, y doblarà como queda dicho, con la diferencia que quando esta doblado, pasarà el pie derecho hasta la $4^{a}$ posicion, pero ha de ser tendiendo las rodillas, y relevando el cuerpo, de manera, que quando llegue à la $4^{a}$ posicion, se ha de encontrar relevado, y firmes las rodillas: dexarà caer el talon derecho, y pasarà el pie izquierdo, empezando à doblar desde la $4^{\mathrm{a}}$ atras, y arrastrando la punta del mismo pie hasta llegar à la $4^{a}$ adelante, continuara con el mismo pie sin detenerse en la $1^{\text {a }}$ posicion; aunque no hay ningun doblado que para ser perfecto no salga de la $1^{a}$ posicion, pero este aunque verdaderamente no sale porque empieza à doblar desde la $4^{\mathrm{a}}$ atras, se supone que sale de la $1^{\mathrm{a}}$, respecto de que es la ultima posicion que pasa: y asi se debe suponer y entender. En estos dos demicupes uno del pie derecho y otro del izquierdo se gastarà el mismo tiempo de un paso de Minue, que son dos compases de musica. Estos demicupés nunca se pueden empezar, sino por el pie derecho, que es el que lleva el compas, siempre que es compás de minue. Para marchar atras, se observaran las mismas reglas, con la diferencia de hacer la quarta atras como antes se hacia adelante: y advirtiendo, que siempre que pase el pie izquierdo debe ser arrastrando.

\section{Sexta leccion del modo de hacer los demicupes de los lados.}

A la derecha: se pondra el caballero en la $1^{a}$ posicion y doblarà como queda dicho; y quando haya doblado, pasarà el pie derecho à la $2^{\mathrm{a}}$, tendiendo la rodilla, y relevado el cuerpo; dexarà caer el talon derecho, y doblando las dos rodillas, el pie izquierdo irà a la $5^{\mathrm{a}}$ arrastrando la punta del pie, y en llegando à la $5^{\mathrm{a}}$ atras, relevarà el cuerpo, y dexarà caer el talon izquierdo, y empezarà à doblar y à marchar del pie derecho pasandole por delante de la $3^{\mathrm{a}}$ y la $1^{\mathrm{a}}$ hasta llegar à la segunda posicion y continuarà asi mismo, para 
marchar à la izquierda. En acabando de marchar à la derecha, queda el pie izquierdo atras en la $5^{\text {a }}$ posicion, luego se cruza el pie derecho doblando desde la $5^{\mathrm{a}}$ posicion atras, y en ella relevarà y doblarà otra vez, y arrastrando el pie izquierdo le llevara hasta la $2^{\mathrm{a}}$ posicion, doblando y arrastrando, y asi continuarà.

\section{Septima leccion, del modo de hacer el paso de minue adelante.}

Se pondrà el caballero en la $1^{\mathrm{a}}$ posicion. Hara un demicupè adelante con el pie derecho, paso de vorea con el izquierdo, de los quales se compone un paso de minue. Pero se ha de advertir, que el paso de vorea se compone de un doblado y dos pasos naturales; y tambien, que un paso de minue consta de dos compases de musica, que son seis partes,y se reparten de este modo: en el primer paso que es el demicupé 2: otros dos en el segundo que es el doblado del paso de vorea; una en el tercero, y otra en el quarto, que componen los dos compases de musica. Para hacer el paso de minue atras seguiran las mismas reglas, gastando el mismo tiempo: y advirtiendo que el paso de minue nunca se ha de empezar con el pie izquierdo, sea el paso adelante, o sea atras, porque el pie derecho debe llevar siempre el compas, y si se empezase con el izquierdo se verificaria lo contrario.

\section{Octava leccion para hacer el paso de minue a la derecha.}

Se pondrà el caballero en la primera posicion; harà un demicupè de lado con el pie derecho hasta llegar à la $2^{\mathrm{a}}$ posicion, y luego empezara el paso de vorea con el izquierdo hasta llegar à la $5^{\mathrm{a}}$ y continuara el paso hasta concluirle. Para hacer el paso de minue à la derecha, no es menester mas que la $2^{\mathrm{a}}$ posicion y la $5^{\mathrm{a}}$ solamente pasan por delante de la $1^{\mathrm{a}}$ à los doblados, que aunque no se quede en ella se supone que el doblado sale siempre de la primera, como se ha dicho.

\section{Nona leccion del paso de minue à la izquierda}

Se pondra el caballero en la $1^{a}$ posicion; hara un demicupè con el pie derecho cruzandole hasta la $5^{\text {a }}$ luego empezara el paso de vorea con el pie izquierdo y le continuarà hasta acabar el paso.

\section{Explicacion del minue.}

\section{Cortesia.}

Pondrà el caballero el pie derecho con la quarta posicion adelante: le pasara por delante à la tercera hasta llegar à la primera; aqui relevarà un poco el cuerpo, y llevarà el pie derecho à la segunda posicion, le dexarà alli quieto, cargando el cuerpo sobre èl, y arrastrando el pie izquierdo y doblando al mismo tiempo la rodilla izquierda baxarà el cuerpo y la cabeza, y el pie izquierdo continuara arrastrando y doblando hasta llegar entre la $3^{\mathrm{a}}$ y la $4^{\mathrm{a}}$, que esta es media posicion. Aqui quedara el cuerpo enteramente baxo, la rodilla izquierda doblada y la derecha firme. Luego le subirá y pondrà en su equilibrio natural, adelantarà el pie derecho hasta la $4^{\mathrm{a}}$ posicion, arrimara el izquierdo detras en la $3^{a} y$ hara un quarto de vuelta sobre la punta del pie derecho sin mover el izquierdo de atras, pondrà este mismo en la $2^{a}$ posicion y empezara à arrastrar el derecho y à doblar la rodilla derecha, baxando al mismo tiempo el cuerpo; $y$ el pie derecho continua arrastrando hasta pasar por delante de la $1^{\mathrm{a}}$ posicion, y llegar à la $4^{\mathrm{a}}$ atras, quedando la misma rodilla doblada, y la izquierda firme con el cuerpo inclinado desde la cintura arriba. Aqui pondra el cuerpo derecho otra vez, y hara un paso de minue a la derecha, quedando en el ultimo el pie izquierdo adelante, dando un quarto de vuelta sobre la izquierda y quedarà al frente de la sala. Tomarà con su derecha la mano izquierda de la señora, advirtiendo que la mano de la señora ha de ir à la parte de arriba: hara paso de minue de frente, dos pasos atras tomando dos quartos de vuelta, sobre la izquierda, soltara la mano de la señora hara un paso de minue à la derecha, dos à la izquierda, dos adelante dando dos 
quartos de vuelta sobre la izquierda, y continuarà con esta misma figura hasta que la señora señale para darle la mano derecha, que la señalarà al segundo paso a la izquierda, ò a lo menos debe señalarselo, y continuara haciendo el otro paso a la izquierda sin volver el cuerpo, y levantara el brazo derecho al tiempo que la señora hace la accion. Luego harà un paso adelante, tomarà la mano de la señora y harà otro paso adelante, haciendo una vuelta entera, soltara la mano, y hara un paso à la derecha quedando con el pie izquierdo adelante en la $4^{a}$ posicion. Luego levantarà el brazo izquierdo, y hara un paso adelante tomara la mano izquierda de la Señora, $y$ haciendo otro paso con vuelta entera, dexara la mano, y harà un paso à la derecha, y continuara con la misma figura como se ha dicho, dando la vuelta del minue. Y en llegando al mismo sitio donde ha dado la mano derecha, y la izquierda harà los dos pasos a la izquierda, sin volver el cuerpo, levantarà los dos brazos, hara dos pasos adelante, el segundo con un quarto de vuelta sobre la derecha, y tomando la mano derecha de la Señora le tomarà la izquierda, pondrà el pie derecho en la $2^{\mathrm{a}}$ posicion, y harà la cortesia como queda arriba explicado.

\section{Reglas particulares para dar las manos.}

Para dar la mano derecha harà el caballero un paso de minué á la izquierda con el movimiento que queda arriba explicado; seguirà el segundo paso à la izquierda, y à la mitad de este que serà al empezar el tercer paso del segundo traerà la mano derecha adelante hasta el borde del vestido, que es la prevencion para el movimiento, y al tiempo de empezar el paso adelante subirà el codo hasta que se conozca, que tendiendo el brazo, queda igual con el hombro. Luego se tiende todo el brazo, despues se doblarà formando un medio circulo, y volviendo un poco la palma de la mano hácia dentro, se toma la mano de la Señora, y con ella se hace media vuelta hasta llegar al lugar opuesto de donde se ha dado la mano. Entonces se suelta la mano de la Señora, y se hace un paso à la derecha todo el qual se gasta para tender el brazo y dejarle caer. En todo este tiempo se tendra vuelta la cabeza sobre la derecha, pues asi se mira siempre a la compañera. Las mismas reglas se observaran para dar la mano izquierda; solo que se tendrà vuelta la cabeza sobre la izquierda; y lo mismo se practicarà para dar las dos manos, solo que entonces se ha de mirar al frente.

\section{Reglas para el portamento de cabeza.}

Luego que el caballero estè al frente de la Sala con su compañera para empezar el minue, volverà la cabeza à la derecha donde esta la Señora. Al poner el pie derecho en la segunda posicion para empezar la cortesia, volvera la cabeza al frente para dar à conocer que la primera cortesia es para el concurso. Despues de esta primera cortesia volverà la cabeza à la derecha, para que se conozca que la segunda cortesia es para la compañera. Se hace el paso de minue à la derecha, y se sigue el paso adelante siempre con la cabeza sobre la derecha hasta llegar al puesto en donde se dexa la mano de la Señora, que es quando empieza la figura del minue con dos pasos à la derecha y luego à la izquierda. Quando se va à la derecha, la cabeza ha de mirar à la izquierda, y seguiràn los dos pasos en la misma disposicion. Luego empiezan los dos pasos à la izquierda: en el primero no moverà el caballero la cabeza; pero en el segundo la volvera sobre la derecha con mucha suavidad: advirtiendo que quando se va sobre la derecha, se ladea el cuerpo à la derecha, y quando à la izquierda, sobre la izquierda, de manera que el cuerpo va siempre en direccion contraria à la de la cabeza. Quando se va adelante se vuelve un poco el cuerpo sobre la derecha, la cabeza del mismo modo y asi se sigue todo el minue, observando para acabar las mismas reglas en orden à la cortesia que se han dicho arriba. 


\section{Reglas para el movimiento delos brazos.}

El movimiento de los brazos consta de tres tiempos: es a saber: relevar el codo, separar los brazos del cuerpo y dexarlos caer, de modo que caigan las manos encima de los bolsillos de la casaca, y la palma de la mano un poco vuelta hacia afuera. En estos tres movimientos se gastan cinco partes de musica. El compas dà al relevar el codo; la otra parte de musica que falta para completar los dos compases, que se invierten en cada paso de minue se completa volviendo los brazos de atras adelante, los quales han de llegar al borde del vestido por delante; y asi va el movimiento del brazo unido con el paso: advirtiendo, que en cada paso de minue estaran los tres tiempos que componen el movimiento del brazo; y se tendrà gran cuidado, que nunca pare el brazo en los movimientos dichos, porque sino faltaria ò sobraria musica, y no podria ir igual con el paso.

\section{Advertencia sobre las cortesias.}

Se debe observar que la cortesia que hace un caballero parado, de paso á derecha y izquierda, al entrar y salir de una sala, no sea ni demasiado despaciosa, ni muy inclinada, que toque en afectacion. Al entrar en una sala no es necesaria mas que una cortesia en el puesto en que se anuncia el sugeto, otra à la persona ò personas que presiden la sala, y alguna inclinacion hacia los demas: Al despedirse una cortesia à las personas que presiden y alguna leve inclinacion à los demas. Las inclinaciones muy baxas se dexan solo para personas Reales, y para los ministros: las demas cortesias se han de hacer con poca inclinacion, y con brevedad natural. Por ultimo se ha de advertir, que ál hacer la inclinacion en la cortesia, no se dexen colgar los brazos hacia adelante con movimiento del hombro, que es feo, y parece dislocacion de brazos, sino que esten entre unidos, y separados muy poco y suavemente del cuerpo con la mayor naturalidad posible.

Real Seminario 12 de setiembre de 1800.

Manuel de Valbuena" 